\title{
A new method for extracting the glottal closure intervals of voiced speech
}

\author{
Tetsuo Funada \\ Faculty of Technology, Kanazawa University, \\ 2-40-20, Kodatsuno, Kanazawa, 920 Japan \\ (Received 30 January 1989)
}

\begin{abstract}
The purpose of the paper is to find out the instant of the glottal opening/closure from the speech signal by a recursive algorithm, and to accurately estimate the characteristics of a vocal tract by using glottal closure covariance analysis. The identification of the glottal closure/opening was achieved by estimating the waveform of the glottal volume flow. A recursive estimation of the glottal volume flow was realized by using a timevarying AR model with an unknown nonwhite input ( $u$-input). In the applications to synthetic vowels generated by the two-mass model, it is shown that the phase of the estimated $u$-input corresponds to that of the glottal volume flow. It is also shown in the experiment for real vowels uttered by male adults that the phase of the estimated $u$ input corresponds to that of the glottal movements which can be confirmed by observing the Electroglottograph (EGG). The glottal closure intervals are determined from the estimated $u$-input, and stable formant frequencies are estimated by covariance analysis which utilizes only the speech during glottal closure intervals. The estimates are compared to the conventional autocorrelation LP analysis. As results of some experiments, we show that the proposed method is effective for the identification of the glottal closure intervals.
\end{abstract}

PACS number: 43. 72. Ar

\section{INTRODUCTION}

It has been well known that the glottal volume flow causes extraneous error in the estimates of the formants for voiced speech. This error may originate from a harmonic structure and a glottal volume flow since the glottal waveform is not an exact pulse train. It has been reported that pitch synchronous covariance analysis is effective for the diminution of the influence on formant estimates caused by the harmonic structure. ${ }^{1,2)}$ However, this analysis method cannot decrease the influence which is given by the waveform of the glottal volume flow. Therefore, it is necessary to develop a pitch synchronous covariance analysis which utilizes only the speech during glottal closure intervals. To identify the intervals, the dependency of LPC prediction error on the position of the analysis frame has been utilized by many researchers so far. ${ }^{3-5}$ ) These methods, however, are some kinds of frameby-frame analysis, and require much computation. It has also been reported that an Electroglottograph (EGG) was used to find out the instant of the glottal opening/closure. ${ }^{6-9)}$ This method requires a twochannel analysis, and it is difficult to implement the method in a speech recognition machine.

The purpose of this paper is to find out the instant of the glottal opening/closure from only speech signals by a recursive algorithm, and to accurately estimate the characteristics of a vocal tract. In the previous paper, ${ }^{10)}$ the author has proposed the estimation of an index to the glottal closure by using the mutual information between a sample point of the speech signal and its autoregressive coefficients. The index can help us estimate the instant of the glottal closure. In this paper, the 
author applies an AR model with an unknown auxiliary nonwhite input to speech signals. ${ }^{11)}$ This model is called "AR model with unknown input" in this paper. The unknown input ( $u$-input) may enable us to estimate the waveform of the glottal volume flow. The instant of the glottal opening/ closure will thereby be determined from the estimated $u$-input, and the characteristics of a vocal tract can be exactly obtained by the proposed pitch synchronous covariance analysis which utilizes only the speech during glottal closure intervals. In addition, the estimated $u$-input may be effectively used for classifying the voiced/unvoiced intervals.

\section{AR MODEL WITH UNKNOWN INPUT}

The production of a voiced sound can be expressed by an AR model. In general, the input of an AR model is assumed to be white. In comparisons of the AR model with the production model of a voiced sound, however, the input of the model (i.e., glottal volume flow) is actually nonwhite (see Fig. 1). Thus the estimated AR model includes undesired characteristics of the glottal volume flow as well as the vocal tract. To separate each characteristic, a time-varying AR model with unknown input can be used. This input is introduced to express the glottal volume flow. Therefore, the estimated AR model may include less information on the glottal volume flow, and give somewhat precise vocal tract characteristics.

The AR model with unknown input can be described as the following equation:

$$
\begin{aligned}
& y(n)=\sum_{i=1}^{p} a_{i}(n) y(n-i)+u(n)+e(n), \\
&(n=0,1,2, \ldots)
\end{aligned}
$$

where $y(n)$ is a sample of speech signals at a $10 \mathrm{kHz}$ sampling rate, $\left\{a_{i}(n)\right\}_{i=1}^{p}$ are the time-varying $\mathrm{AR}$ coefficients, $e(n)$ is a zero-mean Gaussian white noise

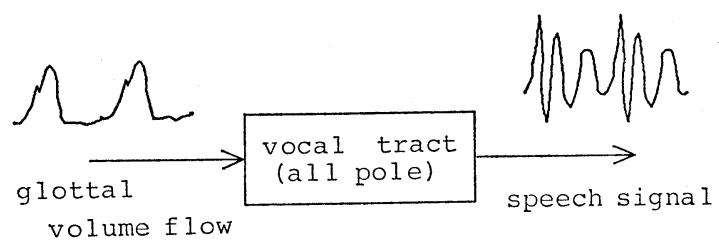

Fig. 1 Production of a voiced sound. with variance $r$, and $u(n)$ is the unknown auxiliary input. The $\mathrm{AR}$ coefficient $a_{i}(n)$ is assumed to be obtained by a stochastically perturbed model:

$$
a_{i}(n+1)=a_{i}(n)+w_{i}(n) \quad(i=1,2, \ldots, p),
$$

where $w_{i}(n)$ is assumed to be a zero-mean Gaussian white noise with variance $q$ for all $i$. The $u$-input $u(n)$ is also assumed to be obtained by the following stochastically perturbed model:

$$
u(n+1)=s \cdot u(n)+w_{p+1}(n),
$$

where $w_{p_{+1}}(n)$ is a zero-mean Gaussian white noise with variance $q$, and $s(<1.0)$ is a positive constant. The parameter $s$ indicates the time factor for smoothing the estimated $u$-input. When $s$ is close to unity, the estimated $u$-input takes much time to reach its stable value. Conversely, when $s$ is set to be nearly zero, the $u$-input in (1) becomes useless since the $u$-input is almost identical with the white noise. The appropriate value of the parameter $s$ will be experimentally determined in Section 3.

Moreover, we assume that $\left\{w_{i}(n)\right\}_{i=1}^{p+1}$ are mutually independent and $r \gg q$. Let

$$
\begin{aligned}
& \boldsymbol{X}(n)=\left(a_{1}(n), \ldots, a_{p}(n), u(n)\right)^{\mathrm{t}} \\
& \boldsymbol{C}(n)=(y(n-1), \ldots, y(n-p), 1)^{\mathrm{t}} \\
& \boldsymbol{W}(n)=\left(w_{1}(n), \ldots, w_{p}(n), w_{p+1}(n)\right)^{\mathrm{t}},
\end{aligned}
$$

then (1); (2) and (3) are rewritten as the following dynamic equation:

$$
\left\{\begin{array}{l}
y(n)=\boldsymbol{C}^{\mathrm{t}}(n) \boldsymbol{X}(n)+e(n) \\
\boldsymbol{X}(n+1)=\boldsymbol{B}_{p+1} \boldsymbol{X}(n)+\boldsymbol{W}(n),
\end{array}\right.
$$

where $\boldsymbol{B}_{p+1}$ is

$$
\boldsymbol{B}_{p+1}=\left(\begin{array}{ll}
\boldsymbol{I}_{p} & 0 \\
0 & s
\end{array}\right)
$$

and $\boldsymbol{I}_{p}$ is the $p \times p$ unit matrix.

The state variable $\boldsymbol{X}(n)$ can be estimated by the following Kalman filter:

$$
\left\{\begin{array}{l}
\boldsymbol{K}(n)=\boldsymbol{P}(n) \boldsymbol{C}(n)\left(\boldsymbol{C}^{\mathrm{t}}(n)+r\right)^{-1} \\
\hat{\boldsymbol{X}}(n \mid n)=\hat{\boldsymbol{X}}(n \mid n-1)+\boldsymbol{K}(n)(y(n) \\
\left.\quad-\boldsymbol{C}^{\mathrm{t}}(n) \hat{\boldsymbol{X}}(n \mid n-1)\right) \\
\hat{\boldsymbol{X}}(n+1 \mid n)=\boldsymbol{B}_{p+1} \hat{\boldsymbol{X}}(n \mid n) \\
\boldsymbol{G}(n)=\boldsymbol{P}(\mathrm{n})-\boldsymbol{K}(n) \boldsymbol{C}^{\mathrm{t}}(n) \boldsymbol{P}(n) \\
\boldsymbol{P}(n+1)=\boldsymbol{B}_{p+1} \boldsymbol{G}(n) \boldsymbol{B}_{p+1}{ }^{\mathrm{t}}+\boldsymbol{Q},
\end{array}\right.
$$

where $\boldsymbol{Q}$ is the $(p+1) \times(p+1)$ covariance matrix of $W(n)$, which is the unit matrix multiplied by $q$, and $\hat{\boldsymbol{X}}(n \mid k)$ is the best estimate of $\boldsymbol{X}(n)$. The last element of $\hat{X}(n \mid k)$ is the estimate of $u$-input at the 


\section{T. FUNADA: EXTRACTION OF THE GLOTTAL CLOSURE INTERVAL}

time instant $n$. This recursion starts with appropriate initial conditions of $\hat{\boldsymbol{X}}(0 \mid-1), \boldsymbol{P}(0)$ and $y(k)$ $(k<0)$.

\section{PERFORMANCE EVALUATION WITH SYNTHETIC VOWELS}

The above algorithm is applied to the synthesized five vowels generated by the Ishizaka-Flanagan's two-mass model. ${ }^{12)}$ For each vowel, only the stiffnesses and the masses of the vocal cord in the model are changed; the stiffnesses and the masses of the vocal tract, however, are not changed. Thus only the pitch is varied without any change of the formant frequencies. The assigned values of the vocal cord parameters are shown in Table 1. The values of BOIN0 are the standard values assigned by Ishizaka and Flanagan. The pitch of BOINO is about $150 \mathrm{~Hz}$. In BION1, only the stiffnesses are increased, so that the pitch becomes higher than BOIN0. Since the masses increase in BOIN2, the

Table 1 The values of the vocal cord parameters and the ranges of pitch variation for the synthesized vowels.

\begin{tabular}{lccrrrr}
\hline & $\begin{array}{c}\text { Mass 1 } \\
{[\mathrm{g}]}\end{array}$ & $\begin{array}{c}\text { Mass 2 } \\
{[\mathrm{g}]}\end{array}$ & $\begin{array}{c}\text { Stiffness } \\
1 \\
{[\mathrm{dyn} / \mathrm{cm}]}\end{array}$ & $\begin{array}{c}\text { Stiffness } \\
2 \\
{[\mathrm{dyn} / \mathrm{cm}]}\end{array}$ & \multicolumn{1}{l}{$\begin{array}{l}\text { Pitch } \\
{[\mathrm{Hz}]}\end{array}$} \\
\hline BOIN 0 & 0.125 & 0.025 & $80,000.0$ & $8,000.0$ & $145-154$ \\
BOIN 1 & 0.125 & 0.025 & $120,000.0$ & $12,000.0$ & $161-170$ \\
BOIN 2 & 0.1875 & 0.0375 & $80,000.0$ & $8,000.0$ & $120-127$ \\
\hline
\end{tabular}

pitch is lower than that of BOIN0.

Typical results from the synthesized vowel (BOIN0) are shown in Fig. 2. The original speech signals and the glottal volume flows, as well as the estimated $u$-inputs, are shown in this figure. The estimated $u$-input was smoothed by using the three point-moving average. In this experiment, the values of $p, q, r, \boldsymbol{P}(0), \hat{\boldsymbol{X}}(0 \mid-1)$ and $s$ were set to $12,0.02,500,100,0.0$ and 0.97 , respectively. These values were experimentally determined. The dependency of the estimated results on the parameter $s$ is also shown in Fig. 2. All the parameters except $s$ are fixed to the same values as described above. In the case of $s=0.99$, it takes about $90 \mathrm{~ms}$ to reach stable estimation. Moreover, the average slope of the estimated $u$-input shows negative during glottal closure intervals. Conversely, in the case of $s=$ 0.95 , the estimation becomes stable in $20 \mathrm{~ms}$ from its beginning, and the estimated $u$-input shows a positive slope during glottal closure intervals. Therefore, in the cases of $s=0.99$ or $s=0.95$, it should be difficult to identify the glottal closure intervals from the estimated $u$-input. Considering these results, the appropriate value of $s$ was assigned to 0.97 in this experiment.

It is observed that the phase of the estimated $u$ input corresponds to that of the glottal volume flow. Just when the glottis closes, however, negative dips have appeared in the estimated $u$-input. These dips seem to be caused by discontinuity of time-derivatives of the volume flow at that instant. In the case of natural vowels, these dips do not appear (see
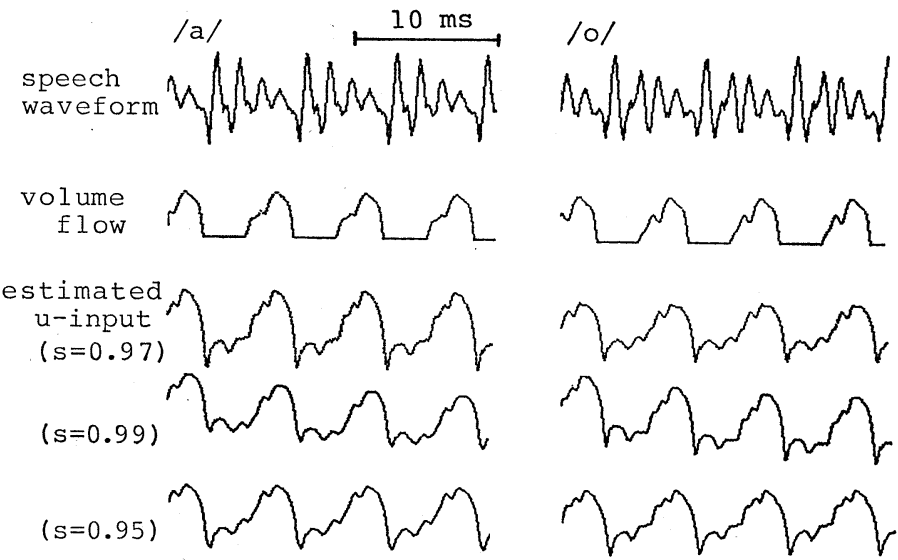

Fig. 2 Examples of estimated $u$-inputs (after applying the three point-moving average) from the synthesized vowels $/ \mathrm{a} /$ and $/ \mathrm{o} /$, the speech signals, and the glottal volume flows. 


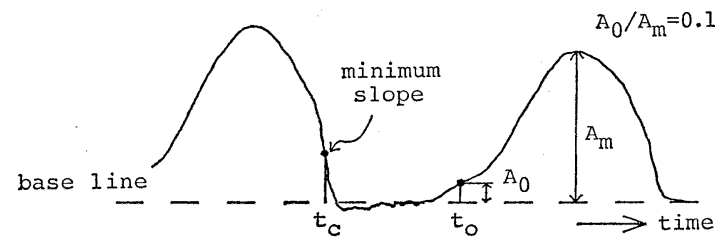

Fig. 3 Determination of the glottal closure instant $t_{\mathrm{c}}$ and opening instant $t_{\mathrm{o}}$.

Fig. 5).

The beginning of a closed interval of the glottis is determined by assigning the instant of the minimum slope of the estimated $u$-input. As shown in Fig. 3, the instant of the glottal opening can be determined at the point where the $u$-input crosses $10 \%$ of the maximum value $A_{\mathrm{m}}$ in a positive slope.

Pitch synchronous covariance analysis, which utilizes only the speech during glottal closure intervals of three consecutive pitch periods, is applied to estimate the formant frequencies. We call this method of analysis "closed phase covariance analysis." The estimated formant frequencies for the same vowels with three different pitches are plotted in column $\mathrm{C}$ of Fig. 4. In column $\mathrm{A}$ of this figure, the formants estimated by the conventional autocorrelation method are shown. The values calculated by the autocorrelation method have a large dispersion among the same vowels with different pitches, compared to the values obtained by the proposed method, i.e., closed phase covariance analysis. Moreover, it turns out that the values calculated by the autocorrelation method considerably differ from the true values shown in column $T$ of Fig. 4. This deviation may primarily be caused by the waveform of the glottal volume flow. The results of these experiments suggest that the estimation of $u$-inputs is effective for identification of the glottal closure intervals.

\section{APPLICATIONS TO NATURAL VOWELS}

The proposed method is applied to natural vowels uttered by male adults at a moderate intensity. Figure 5 shows typical results of vowels /a/ and /e/ in a stationary portion. The speech signals, estimated $u$-inputs, and Electroglottograph (EGG) are shown in this figure. The instant at the maximum positive slope of the EGG indicates the glottal closure. ${ }^{7)}$ In this experiment, the location of the microphone pickup was $30 \mathrm{~cm}$ from the lips. Therefore, the minimum slope of $u$-input is located about 1 $\mathrm{ms}$ from the maximum slope of the EGG. This discrepancy is caused by the delay of sound transmission from the glottis to the microphone pickup.

Figure 6 shows the estimated first three formant

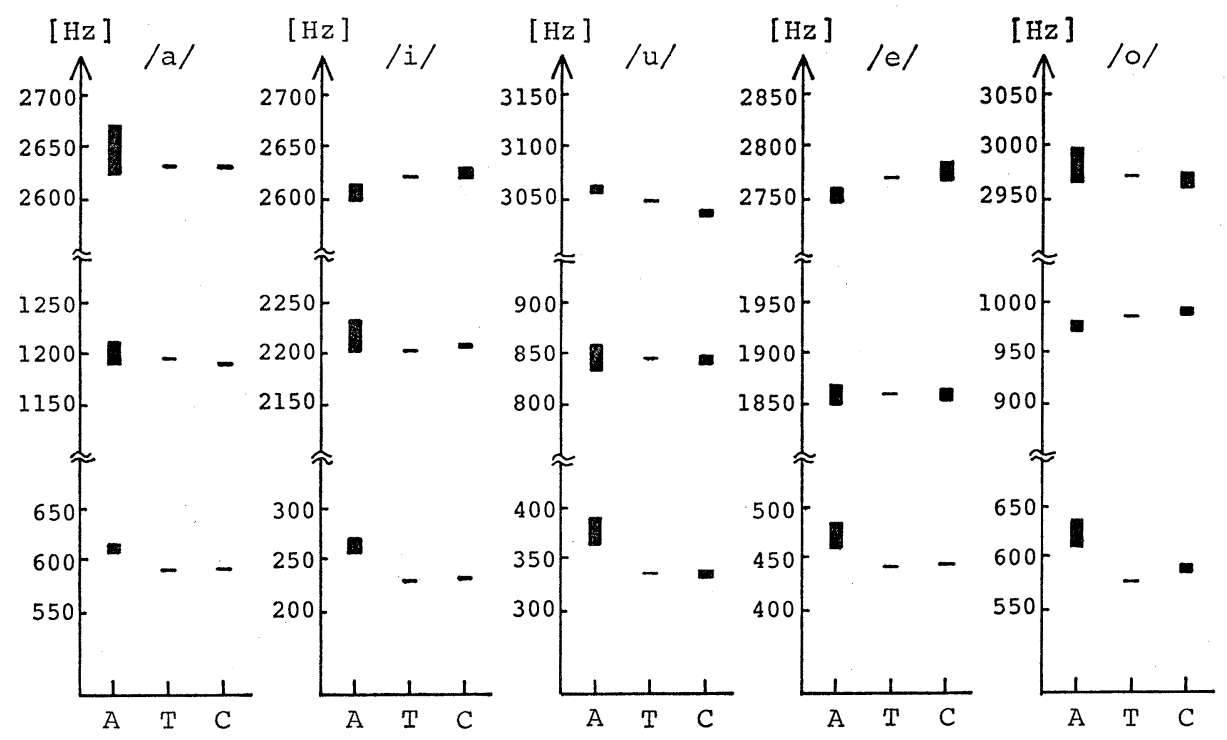

Fig. 4 Estimated first three formant frequencies [Hz] for the same vowels with three different pitches (A: autocorrelation, $\mathrm{C}$ : closed phase, $\mathrm{T}$ : true value). 


\section{T. FUNADA: EXTRACTION OF THE GLOTTAL CLOSURE INTERVAL}

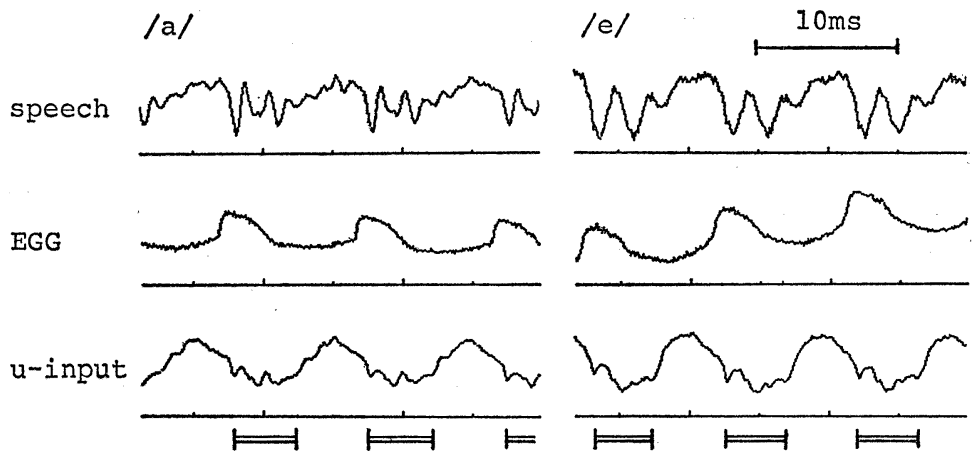

Fig. 5 Examples of estimated $u$-inputs (after applying the three point-moving average) from natural vowels $/ \mathrm{a} /$ and $/ \mathrm{e} / \mathrm{.}(\mathrm{l}=\mathrm{l}$ : closed intervals extracted by the proposed algorithm shown in Fig. 4.)
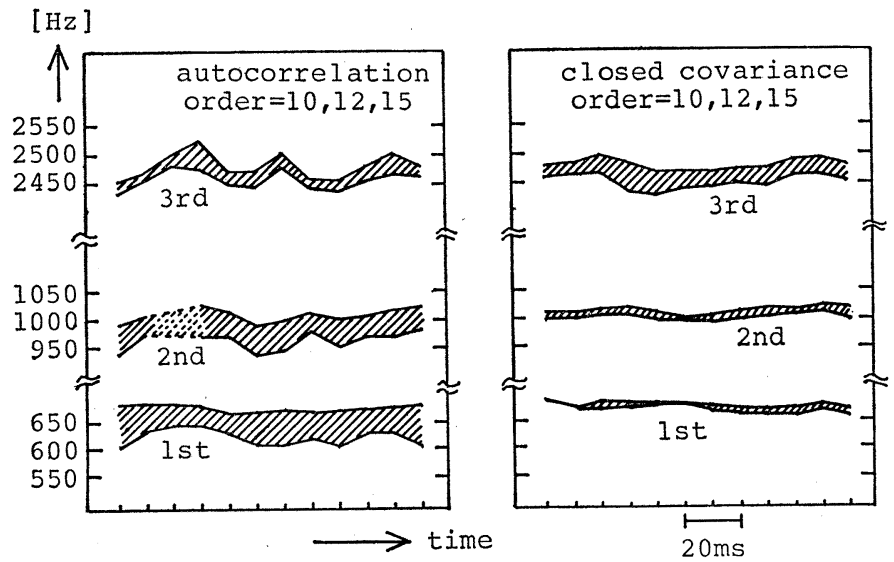

Fig. 6 The order dependency of estimated formant frequencies of a natural vowel on the methods applied. The shaded areas are the total variations of the estimates with three analysis orders, 10,12 and 15. The dotted lines indicate that the method can not extract formant frequency.

frequencies by the closed phase covariance, in which the glottal closure intervals were determined by the algorithm described in Section 3. The shaded area represents the total variation of the estimated formant frequency with three different analysis orders, i.e., 10, 12 and 15 . The results were calculated by a pitch synchronous autocorrelation method, which uses all of the speech during three pitch periods. These are also shown in this figure. Note that stable formant frequencies are obtained irrespective of their analysis order in the case of the closed phase covariance analysis. In particular, the first formant is stable since the influence of glottal volume waveform is significant in a low frequency range (first formant).

\section{DISCUSSION}

In order to compare the proposed method with conventional ones, two methods are chosen in this paper. The first is Wong's method ${ }^{2)}$ which uses only the speech samples during glottal closure intervals. In this method, the minimum of the normalized mean square error derived from the covariance LP analysis is utilized for locating the instants of glottal closure/opening. The second is the Sample-Selective Linear Prediction (SSLP). ${ }^{5}$ ) This employs only relevant samples to accurate formant estimation irrespective of glottal opening/ 
(a) synthesized vowe 1

speech waveform

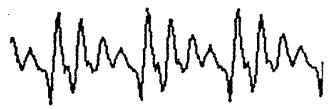

(g 1 otta 1 volume flow)

estimated u-input

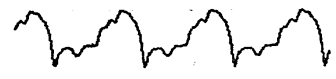

norma 1 ized residual signal

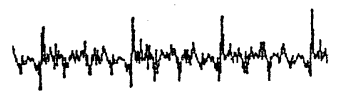
normalized mean
square error

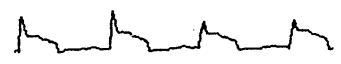

(b) natural vowel

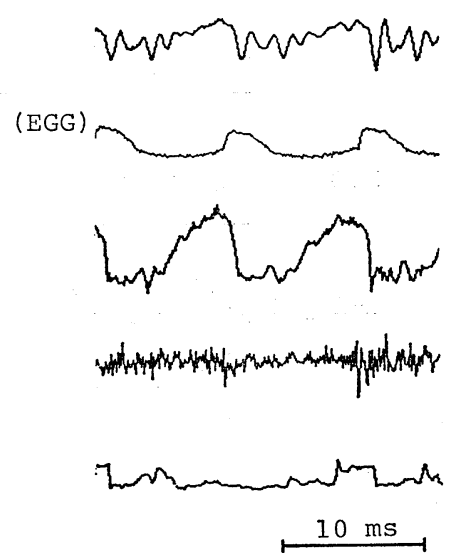

Fig. 7 Estimated $u$-inputs by the proposed method, the normalized mean square errors by Wong's method, the normalized residual signals for executing SSLP from (a) synthesized vowel /a/ and (b) natural vowel /a/.

closure. The selection of the relevant samples is achieved by referring to the normalized residual signals derived from the conventional covariance analysis. Once after determining glottal closure intervals or selecting the relevant samples, these two methods are almost the same as the proposed method. Thus the simplicity or reliability for determining the intervals of glottal closure or selecting the relevant samples are the main factors for comparison among these methods.

Figure 7 shows the examples of the $u$-inputs estimated by the proposed method, the normalized mean square errors by Wong's method, and the normalized residual signals for executing SSLP from synthesized vowel /a/ (BOIN0) and natural vowel /a/. For the synthesized vowel, all of the three methods show stable patterns and can extract the intervals of glottal closure and select the relevant samples rather easily. On the other hand, although a regular pattern is obtained from the estimated $u$ input of the natural vowel, the result of SSLP will be much dependent on the value of the threshold $\left.\theta,{ }^{5}\right)$ which determines the selection of the relevant samples. Moreover, the correct intervals of glottal closure cannot be extracted from the results of Wong's method since the minimum values of the normalized mean square error exhibit an irregular pattern. Thus we can conclude that the determination of the intervals of glottal closure by the

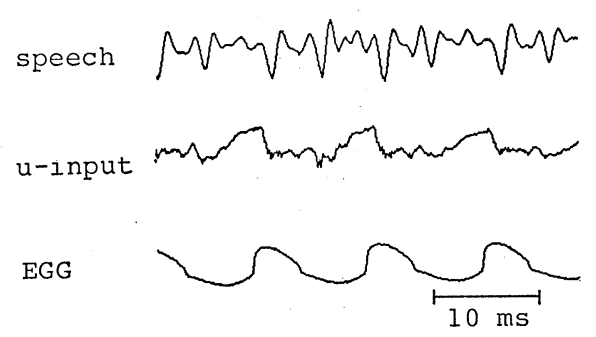

Fig. 8 Example of the estimated $u$-input from natural vowel $/ \mathrm{o} /$.

proposed method is simple and reliable compared with the other two methods.

Figure 8 shows the speech wave /o/, the estimated $u$-input, and the EGG. Although the pitch period of the speech wave is unclear by using visual inspection, the estimated $u$-input shows rather explicit periodicity. Thus $u$-input can be utilized to simplify voiced/unvoiced classification and to improve the pitch-detection score. As the pitch/intensity increases, the instant of the glottal closure/opening tends to be unclear. In five vowels, the estimated $u$-inputs of the vowel /i/ show rather unclear glottal closure/opening, which may be caused by the fact that the first formant is low. We have not yet examined the results for female vowels. 


\section{T. FUNADA: EXTRACTION OF THE GLOTTAL CLOSURE INTERVAL}

\section{CONCLUSION}

An estimation of the glottal volume flow and the characteristics of a vocal tract was achieved by using a time-varying AR model with unknown input. In applications to synthetic vowels generated by the two-mass model, the phase of the estimated $u$-input is shown to correspond to that of the glottal volume flow. Moreover, it was observed from real vowels uttered by male speakers that the phase of the estimated $u$-input corresponds to that of the glottal movement which can be confirmed by observing the Electroglottograph. Therefore, the glottal closure intervals were determined from the estimated $u$ inputs, and stable formant estimates were obtained by using the closed phase covariance analysis. As the further problem, the proposed method should be improved for speech of low formant frequency and high pitch.

\section{ACKNOWLEDGMENT}

The authors would like to thank the reviewers for their suggestions and cooperation in the arrangement of this paper.

\section{REFERENCES}

1) H. W. Strube, "Determination of the instant of glottal closure from the speech wave," J. Acoust. Soc. Am. 56, 1625-1629 (1974).

2) D. Y. Wong, J. D. Markel, and A. H. Gray, "Least squares glottal inverse filtering from the acoustic speech waveform," IEEE Trans. Acoust. Speech Signal Process. ASSP-27, 350-355 (1979).

3) L. R. Rabiner, B. S. Atal, and M. R. Sambur,
"LPC prediction error-Analysis of its variation with the position of the analysis frame," IEEE Trans. Acoust. Speech Signal Process. ASSP-25, 434-442 (1977).

4) H. Kawahara, K. Tochinai, and K. Nagata, "On the linear predictive analysis using a small analysis segment and its error evaluation," J. Acoust. Soc. Jpn. (J) 33, 470-479 (1977) (in Japanese).

5) Y. Miyoshi, K. Yamato, R. Mizoguchi, M. Yanagida, and O. Kakusho, "Analysis of speech signals of short pitch period by a Sample-Selective Linear Prediction," IEEE Trans. Acoust. Speech Signal Process. ASSP-35, 1233-1240 (1987).

6) A. K. Krishnamurthy and D. G. Childers, "Twochannel speech analysis," IEEE Trans. Acoust. Speech Signal Process. ASSP-34, 730-743 (1986).

7) D. G. Childers and J. N. Larar, "Electroglottography for Laryngeal function assessment and speech analysis," IEEE Trans. Bio-Med. Electron. BME-31, 807-817 (1988).

8) S. Imaizumi and S. Kiritani, "Effects of speaking rate on formant trajectories and their inter-speaker variations," 2nd Jt. Meet. ASA and ASJ, NN18, 19 (1988).

9) W. Hess and H. Indefrey, "Accurate time-domain pitch determination of speech signals by means of a Laryngograph," Speech Commun. 6, 55-68 (1987).

10) T. Funada, "On the short time estimation of speech spectrum," Proc. Int. Conf. Digital Signal Processing, 1981, 481-488 (1981).

11) T. Funada, "Speech analysis using a time-varying ARX model for separating the source-tract coupling of vowels," 2nd Jt. Meet. ASA and ASJ, J8, 1-8 (1988).

12) K. Ishizaka and J. L. Flanagan, "Synthesis of voiced speech from a two-mass model of the vocal cords," Bell Syst. Tech. J. 51, 1233-1268 (1972). 